\title{
Long-lasting stable disease with mTOR inhibitor treatment in a patient with a perivascular epithelioid cell tumor: A case report and literature review
}

\author{
EZEQUIEL FLECHTER ${ }^{1}$, YANIV ZOHAR $^{2}$, LUDMILA GURALNIK $^{3}$, MARIA PASSHAK $^{1}$ and GIL BAR SELA ${ }^{1}$ \\ ${ }^{1}$ Division of Oncology; ${ }^{2}$ Pathology Institute; ${ }^{3}$ Computed Tomography Unit, Radiology Department, \\ Rambam Health Care Campus and Faculty of Medicine, Technion-Israel Institute of Technology, Haifa 31096, Israel
}

Received March 17, 2015; Accepted April 4, 2016

DOI: $10.3892 / \mathrm{ol} .2016 .5231$

\begin{abstract}
Perivascular epithelioid cell tumor (PEComa) of the small intestine is extremely rare, and there is no established treatment at the present time. In $10 \%$ of patients with PEComas, genetic alterations of tuberous sclerosis complex have been reported. These genetic alterations activate mechanistic target of rapamycin (mTOR) in AMP-activated protein kinase and Ras/mitogen-activated protein kinase pathways, resulting in high mTOR activity. Since 2007, several cases of treatment with mTOR inhibitors in advanced PEComa have been reported. The current study presents the case of a patient with small bowel PEComa that metastasized to the brain and lungs. Following resection of the brain metastasis, the patient was treated with everolimus, a mTOR inhibitor, resulting in improvement if the patient's quality of life and a long period of stable disease. In conclusion, the use of mTOR inhibitors as a first-line treatment option in advanced PEComa patients appears to be reasonable, according to the increasing evidence from data observed from reported cases with this rare malignancy.
\end{abstract}

\section{Introduction}

Perivascular epithelioid cell tumor (PEComa) was recognized by the World Health Organization in 2002 as 'mesenchymal tumors composed of histologically and immunohistochemically distinctive perivascular epithelioid cells' (1). PEComa exhibits immunoreactivity for HMB-45, melan-A, actin, microphthalmia-associated transcription factor and desmin, and possesses smooth muscle and melanocytic components (2). According to PubMed (www.ncbi.nlm.nih. gov/pubmed), 100 cases of PEComa have been reported in

Correspondence to: Dr Gil Bar Sela, Division of Oncology, Rambam Health Care Campus and Faculty of Medicine, Technion-Israel Institute of Technology, PO Box 9602, Haifa 31096, Israel

E-mail: g_barsela@rambam.health.gov.il

Key words: everolimus, mTOR inhibitor, PEComa, literature review the English literature with various primary sites. The largest series of 35 cases was reported by Doyle et al (3) in 2013. A total of 20 patients out of the 35 were gastrointestinal in origin, 13 patients developed metastasis to various sites, and 5 patients succumbed to advanced disease (3).

In $10 \%$ of PEComas cases, genetic alterations of tuberous sclerosis complex (TSC), due to losses of 9q34 (TSC1) or 16q13.3 (TSC2), have been reported (4). These genetic alterations activate mechanistic target of rapamycin (mTOR) in AMP-activated protein kinase and Ras/mitogen-activated protein kinase pathways, resulting in high mTOR activity (5). This leads to a lack of regulation of cell proliferation, migration and differentiation (6). Since 2007, several cases of treatment with mTOR inhibitors in advanced PEComa have been described (7-12).

The current case presents a patient with small bowel PEComa that metastasized to the brain and lungs. Following resection of the brain metastasis, the patient was treated with everolimus, a mTOR inhibitor, resulting in improvement in the patient's quality of life and a long period of stable disease.

\section{Case report}

A 35-year old woman with morbid obesity, but without any comorbidity, was admitted to The Emek Medical Center (Afula, Israel) in October 2010 due to acute abdominal pain. Abdominal computed tomography (CT) demonstrated small bowel obstruction. An emergency, explorative laparotomy was performed with resection of the intestinal mass. Pathology conducted on the resected mass provided a diagnosis of PEComa. A subsequent chest CT revealed the presence of multiple lung nodules, which were diagnosed as metastases of PEComa by pathology on samples taken using fine-needle biopsy.

The patient was lost to follow-up until April 2011 when she was admitted The Emek Medical Center due to severe headaches and blurred vision. Brain magnetic resonance imaging (MR750w 3.0T; GE Healthcare, Milwaukee, WI, USA) demonstrated the presence of a posterior brain tumor (Fig. 1). Posterior craniotomy and resection of the tumor was performed. The pathological report diagnosed the tumor as metastatic PEComa identical to the small bowel origin (Fig. 2). 


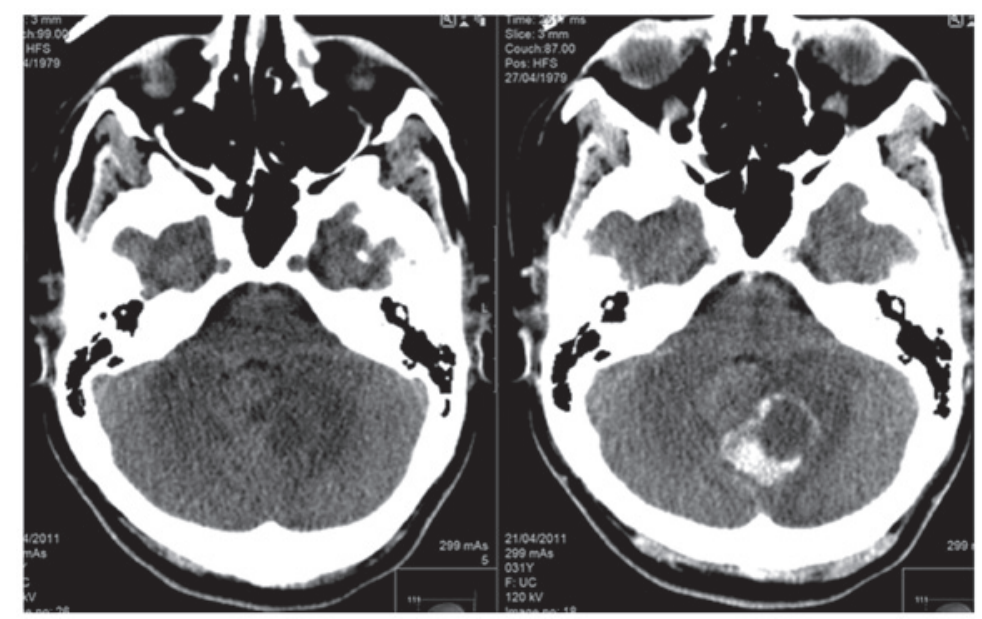

Figure 1. Brain magnetic resonance imaging at the posterior fossa with contrast medium (IV gadolinium) injection in a patient with perivascular epithelioid cell tumor of the small bowel. In the left lobe of the cerebellum, a hypodense peripherally-enhanced lesion with prominent edema and mass effect is observed.

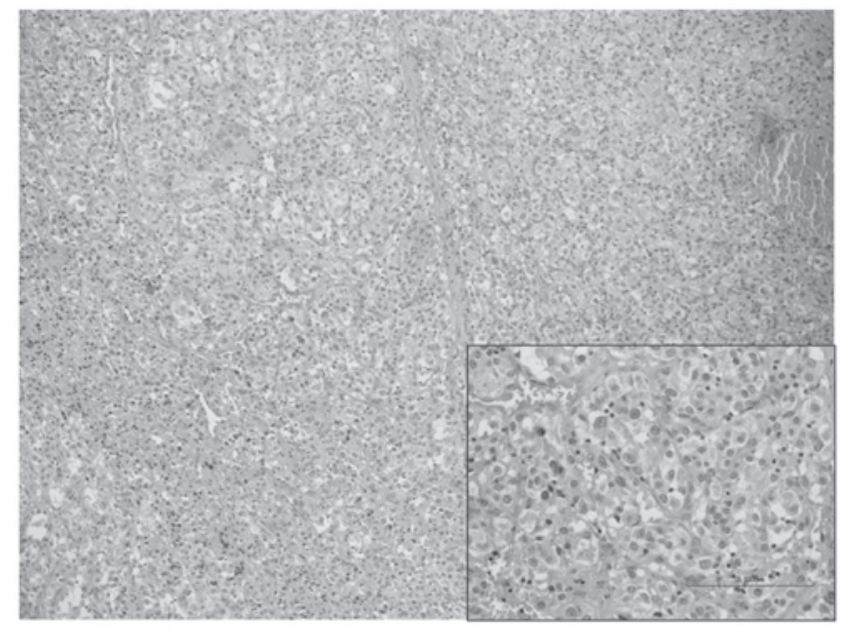

Figure 2. Histology of the brain metastasis. The tumor is composed of packed nests of epithelioid cells with pink granular or partially clear cytoplasms (hematoxylin and eosin staining; x100 magnification, inset x400 magnification).

A

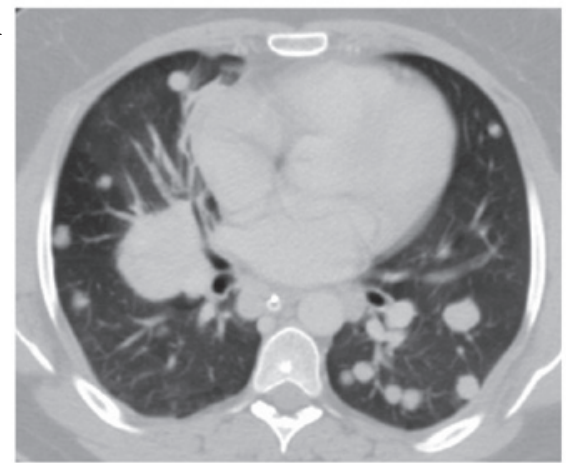

B

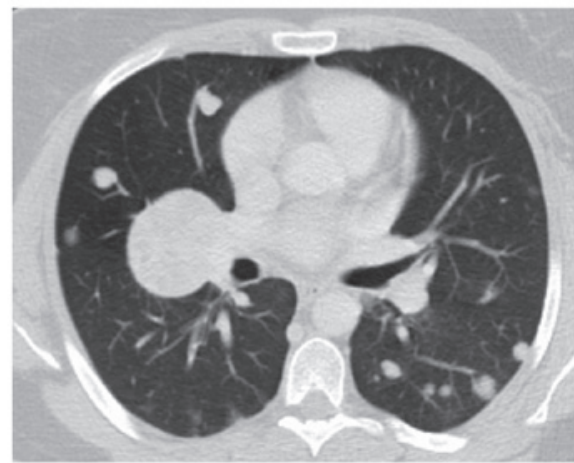

Figure 3. (A) First slice of abdominal CT beneath the bronchus intermedius $\sim 1$ year following the diagnosis of brain metastasis. A large right hilar mass and multiple bilateral pulmonary nodules representing metastatic spread are observed. (B) Thoracic CT beneath the bronchus intermedius 2 months subsequent to the patient beginning treatment with everolimus. The right hilar mass has not altered, but the majority of the pulmonary nodules have decreased in size. No novel nodules are observed. CT, computed tomography.

The patient had an appointment with an oncologist (Rambam Health Care Campus, Haifa, Israel) for the first time in January 2012. The patient's Eastern Cooperative Oncology Group (ECOG) performance status (13) was 2, and she suffered from dyspnea, abdominal pain and weakness. In addition, the patient used a wheelchair and nasal oxygen.

A CT scan for a systemic evaluation demonstrated multiple bilateral lung metastases (Fig. 3A), without evidence of brain 


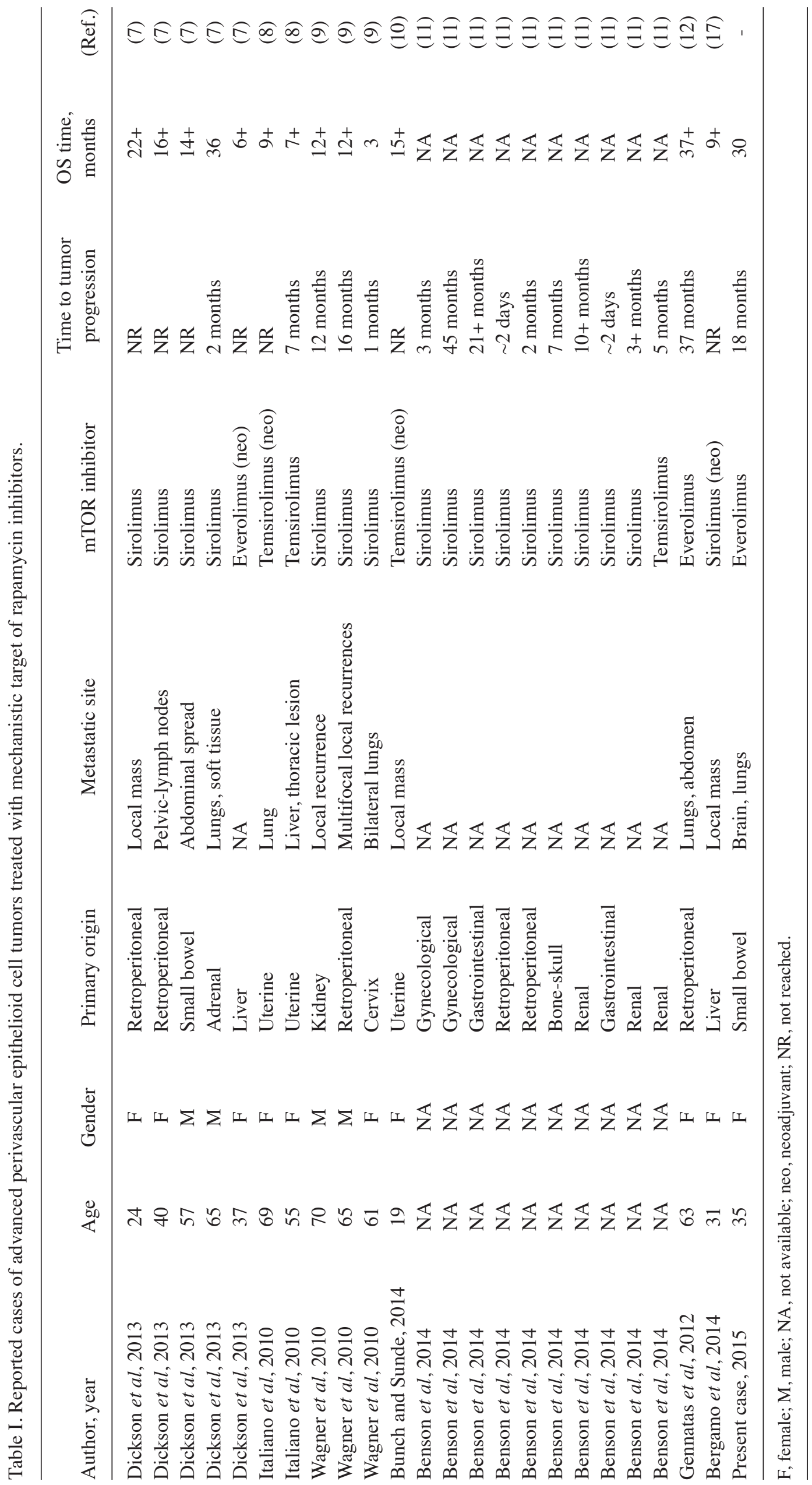


or abdominal recurrence. In May 2012, the patient was administered with a mTOR inhibitor as a first-line treatment for metastatic disease (10 $\mathrm{mg}$ oral everolimus once daily). Following two months of treatment, improvement in the patient's lung metastases (Fig. 3B), quality of life and symptoms was observed. All symptoms of dyspnea, abdominal pain and weakness were improved, and the patient was able to stop using the wheelchair. Radiological evaluations revealed stable disease under continued treatment with everolimus that lasted for 18 months. During this period, the patient was asymptomatic and had an ECOG performance status of 0 . The patient was followed up every two months, and follow-up appointments consisted of history and physical evaluation, cell blood count, blood biochemistry and CT.

In November 2013, the patient complained of right pelvic pain radiating down the right leg, with ipsilateral low extremity lymphedema. A CT scan revealed the presence of novel masses above the right knee and in the pelvic soft tissue, and the already diagnosed lung lesions. Biopsy from the leg mass demonstrated that the pathology was that of metastatic PEComa.

The patient was started on second-line therapy with doxorubicin ( $75 \mathrm{mg} / \mathrm{m}^{2}$ every 21 days for 4 cycles). A response was observed two months later in the soft tissue masses, with reduction of the leg lymphedema, but the disease progressed in the lungs with novel metastases observed five months subsequent to treatment. The patient succumbed to the disease in January 2015. Written informed consent was obtained from the patient's family for the publication of this study.

\section{Discussion}

Primary PEComa of the small intestine is rare and has been reported in only a few cases $(7,14,15)$. The current patient was initially diagnosed with small bowel PEComa with metastatic disease to the lungs, and possibly to the brain, although the brain metastases were only identified four months later when the patient became symptomatic. Brain metastases from PEComa are extremely rare and have only been reported in two additional cases $(14,16)$.

Until the era of mTOR inhibitors, the only option for the treatment of metastatic disease was chemotherapy. However, no formal recommendations were provided for first-line chemotherapy, and treatment strategies differed between reported cases. Based on the genetic alterations of the tuberous sclerosis complex that was identified in certain patients with PEComas (6), resulting in high mTOR activity, several cases reported treatment of this type of disease with mTOR inhibitors. These cases and the current case are summarized in Table I. Italiano et al (8) reported a radiological complete response following neoadjuvant treatment with temsirolimus as a first-line treatment in a patient with lung metastasis, and a partial response in another patient with cardiac metastasis as a second-line treatment following combination chemotherapy. Wagner et al (9) reported three cases treated with sirolimus either as a first-line treatment leading to stable disease (two cases), or as a second-line treatment with a partial response (one case). Neoadjuvant treatment with temsirolimus for primary uterine PEComa in a young female patient (10) and with sirolimus for a patient with primary liver PEComa (17) resulted in partial response and no recurrence following surgery. Dickson et al (7) also reported complete response or prolonged partial response in 4 out of 5 cases with abdominal PEComas. These patients were treated with rapamycin, sirolimus and everolimus.

The largest case series was reported by Benson et al (11) with 10 cases treated at the Royal Marsden Hospital (London, UK). In that study, a partial response was observed in 5 out of 7 assessed patients and symptomatic responses were documented in 7 out of 10 patients. The 1-year survival rate for this series of patients was calculated at $78.8 \%$ with a median overall survival time of 2.4 years (95\% confidence interval, 0.3-4.5 years) and a median follow-up time of 1.9 years.

Treatment with everolimus was reported in two cases only: As a neoadjuvant treatment in 1 patient with primary liver PEComa, which lead to a good partial response allowing the patient to undergo surgery (7); and in 1 patient with primary retroperitoneal PEComa that had metastasized to the lungs, which lead to a complete response in the lung lesions and a partial response in the abdominal mass (12). In the current case, treatment with everolimus lead to stable disease in the patient for 18 months, with symptomatic relief and improvement in the quality of life of the patient for a disease that had rapidly progressed prior to this treatment.

In conclusion, treatment with mTOR inhibitors as a first-line treatment option in advanced PEComa patients appears reasonable, according to the increasing amount of data reported by cases that present this rare malignancy.

\section{References}

1. Folpe AL: Neoplasms with perivascular epithelioid cell differentiation (PEComas). In: World Health Organization Classification of Tumors: Pathology and Genetics of Tumors of Soft Tissue and Bone. Fletcher CDM, Unni KK and Mertens F (eds). IARC Press, Lyon, pp221-223, 2002.

2. Martignoni G, Pea M, Reghellin D, Zamboni G and Bonetti F: PEComas: The past, the present and the future. Virchows Arch 452: 119-132, 2008.

3. Doyle LA, Hornick JL and Fletcher CD: PEComa of the gastrointestinal tract: Clinicopathologic study of 35 cases with evaluation of prognostic parameters. Am J Surg Path 37: 1769-1782, 2013.

4. Hornick JL and Fletcher CD: PEComa: What do we know so far? Histopathology 48: 75-82, 2006.

5. Napolioni V, Moavero R and Curatolo P: Recent advances in neurobiology of Tuberous Sclerosis Complex. Brain Dev 31: 104-113, 2009.

6. Kenerson H, Folpe AL, Takayama TK and Yeung RS: Activation of the mTOR pathway in sporadic angiomyolipomas and other perivascular epithelioid cell neoplasms. Hum Pathol 38: 1361-1371, 2007.

7. Dickson MA, Schwartz GK, Antonescu CR, Kwiatkowski DJ and Malinowska IA: Extrarenal perivascular epithelioid cell tumors (PEComas) respond to mTOR inhibition: Clinical and molecular correlates. Int J Cancer 132: 1711-1717, 2013.

8. Italiano A, Delcambre C, Hostein I, Cazeau AL, Marty M, Avril A, Coindre JM and Bui B: Treatment with the mTOR inhibitor temserolimus in patients with malignant PEComa. Ann Oncol 21: 1135-1137, 2010.

9. Wagner AJ, Malinowska-Kolodziej I, Morgan JA, Qin W, Fletcher CD, Vena N, Ligon AH, Antonescu CR, Ramaiya NH, Demetri GD, et al: Clinical activity of mTOR inhibitor with sirolimus in malignant perivascular epithelioid cell tumors: Targeting the pathogenic activation of mTORC1 in tumors. J Clin Oncol 28: 835-840, 2010.

10. Bunch K and Sunde J: Fertility sparing treatment of a malignant uterine perivascular epithelioid cell tumor: A case report. Gynecol Oncol Case Rep 8: 14-16, 2014.

11. Benson C, Vitfell-Rasmussen J, Maruzzo M, Fisher C, Tunariu N, Mitchell S, Al-Muderis O, Thway K, Larkin J and Judson I: A retrospective study of patients with malignant PEComa receiving treatment with sirolimus or temsirolimus: The royal marsden hospital experience. Anticancer Res 34: 3663-3668, 2014. 
12. Gennatas C, Michalaki V, Kairi PV, Kondi-Paphiti A and Voros D: Successful treatment with the mTOR inhibitor everolimus in a patient with perivascular epithelioid cell tumor. World J Surg Oncol 10: 181, 2012.

13. Oken MM, Creech RH, Tormey DC, Horton J, Davis TE, McFadden ET and Carbone PP: Toxicity and Response Criteria of the Eastern Cooperative Oncology Group. Am J Clin Oncol 5: 649-655, 1982

14. Agaimy A and Wünsch PH: Perivascular epithelioid cell sarcoma (malignant PEComa) of the ileum. Pathol Res Pract 202:37-41,2006.
15. Yanai H, Matsuura H, Sonobe H, Shiozaki S and Kawabata K: Perivascular epithelioid cell tumor of the jejunum. Pathol Res Pract 199: 47-50, 2003.

16. Parfitt JR, Keith JL, Megyesi JF and Ang LC: Metastatic PEComa to the brain. Acta Neuropathol 112: 349-351, 2006.

17. Bergamo F, Maruzzo M, Basso U, Montesco MC, Zagonel V, Gringeri E and Cillo U: Neoadjuvant sirolimus for a large hepatic perivascular epithelioid cell tumor (PEComa). World J Surg Oncol 12: 46, 2014. 